\title{
A preliminary investigation of textile fibers in smothering scenarios and alternative legitimate activities
}

\begin{abstract}
Was this group of target fibers transferred during a criminal action? Is it possible that it was transferred during another legitimate activity? Acquiring knowledge about the activity, whether legitimate or criminal, leading to the transfer of a group of fibers is a recurring challenge encountered throughout the evidence interpretation process. Trace evidence such as fibers may assist with generating a reasoning of the activity which produced the trace, but this assumes that one already has a thorough understanding of the transfer phenomenon. How to generate and then use such relevant knowledge? What are the influencing parameters and which ones should (or can) be controlled?

The present work focuses on homicides by smothering using a pillow and the transfer of fibers on the face of the victim in such events. A legitimate activity - represented by a night's sleep on a pillow was also investigated since such legitimate, alternative explanations concerning the presence of fibers could likely be formulated by the suspect.

The number and distribution of fibers transferred onto the victim's face in either scenario were investigated and interpreted using a Bayesian approach. Results showed that the shedding capacity of the pillowcase strongly impact the number of fibers recovered. The nature of the action, modus operandi and amount of friction could not be excluded in their influence on the transfer of fibers. Finally, likelihood ratios indicating the number of fibers recovered from the victim's face can provide relevant information by supporting either criminal or legitimate proposition (i.e., smothering or night's sleep scenario).
\end{abstract}

\section{Keywords}

\author{
1:1 taping \\ Fiber distribution \\ Fiber transfer \\ Forensic interpretation \\ Trace evidence
}




\section{Introduction}

Textile fibers can be recovered in criminal cases involving intense contact between individuals engaged in physical or sexual assaults, brawls and particularly homicides. For years now, studies highlight that fibers from the perpetrator are transferred onto the victim during the criminal action and conversely [1-4]. The number of textile fibers recovered depends notably on the characteristics of the different substrates (shedding capacity of the donor and structure of the recipient), the type of contact and the phenomenon of persistence [3-7]. These are parameters that should be discussed when reasoning at the activity level [8-10].

When a group of target fibers recovered on the victim's clothes shares common physical and chemical characteristics with a questioned fabric, it is possible to link the fabric to the criminal action with a certain degree of uncertainty. These fiber concordances represent potentially important evidence if the suspect denies any contact with the victim, especially in cases where no DNA trace is available. However, the value of such evidence for the investigation could become very low when the suspect claims having justified and legitimate contact with the victim shortly before the alleged crime. This is a common situation, typically encountered in domestic violence where those involved know each other or even live together. Proposing an alternative activity to explain the presence of fibers also requires consideration of the transfer phenomenon and persistence of fibers. Therefore, reasoning needs to take place at the activity level. Many have already studied transfer and persistence of fibers in various scenarios [1,4,11-17] and, with the exception of transfer on car seats [18-20], most studies have systematically considered simplified activities rather than realistic scenarios [1,4,17,21-24]. Moreover, to the knowledge of the authors, no transfer study has yet been carried out based on legitimate alternative activities involving contact close to criminal actions.

Let us consider a case where a healthy middle age female was found dead on her bed. During the investigation, a group of 170 target orange cotton fibers was collected from her face that were not differentiated from the victim's pillowcase. Although the victim's husband claims he found his wife dead when he woke up in the morning, the prosecutor suspects the husband to have smothered the victim with her pillow during the night.

Legal medicine is frequently requested first in determining the cause of death. However, in smothering cases involving a pillow, it's difficult to detect characteristic signs of asphyxia. Usually, there is absence of any characteristic marks (e.g. congestion, abrasion or petechiae) unless the victim is able to defend him/herself from the assailant [25-30]. It is problematic as typical smothering victims tend to be very young, very old, disabled, or incapacitated by restraints such as illness or drugs [28-30]. In such complex situations, it may be interesting to consider trace evidence such as textile fibers. However, is it more relevant to observe 170 orange cotton fibers on victim's face in the prosecution's scenario (claiming death by smothering) or rather in the account provided by the defendant (natural death)? Without any data, it might be difficult to offer relevant and significant contributions supporting either scenario. Although the evidence in the present case focused only on fibers found on the victim face, it is important to note that fibers can also be recovered from the respiratory tract such as the nose and mouth cavity [31]. To this date, no study which investigates the transfer of fibers in smothering/night's sleep scenarios has been found.

To overcome the lack of information in this field, the present work focuses on two main aspects: first, five volunteers were asked to perform various night's sleep and smothering simulations with different pillowcases which were selected mainly based on their shedding capacity. This allowed for investigating whether the transfer of fibers was variable - based on the number and the localization of fibers on the face - depending on the activity. The consideration of fiber localization is innovative as little research has investigated this particular parameter except in case reports [32-36]. It should be noted that, in the present article, the persistence of fibers was not investigated since it assumes that fibers were already collected at the crime scene and that the victim was not moved or manipulated 
after discovery. In such circumstances, losses can be considered negligible. Secondly, a Bayesian interpretation of the data is proposed as it is crucial to assess the evidential value of a fiber distribution in the light of the activities formulated by the prosecution and defense [37,38]. The knowledge provided in this work will assist the practitioners for the investigation of smothering caseworks and help in assessing the evidence at the activity level.

\section{Materials and Methods}

\subsection{Volunteers}

Five individuals - two men and three women - volunteered to participate in the simulations of this study (Table 1). Each volunteer presented various morphological characteristics, haircuts, facial hair (from "clean-shaven" to "stubble”) and lifestyles (make-up, facial cream and care, etc.). To record the physical characteristics of the volunteers at the moment of the experiment, a questionnaire was filled out prior to each simulation. Details about legitimate simulations were also sought in the questionnaire (see section 2.3).

\begin{tabular}{lllll}
\hline & & VoLUNTEERS (N=5) & \\
\hline Number & Gender & Age & Facial hair & Facial care \\
\hline 01 & Male & 23 & Clean-shaven & None \\
02 & Female & 30 & None & None \\
03 & Female & 27 & None & Night cream \\
04 & Male & 25 & Stubble & None \\
05 & Female & 21 & None & None \\
\hline
\end{tabular}

Table 1. Volunteer characteristics.

\subsection{Pillowcases}

Simulations were performed using two different brands and models of pillowcases:

1. An orange pillowcase produced by Maddison; model Perka; size $60 \mathrm{~cm} \times 60 \mathrm{~cm}$. The fabric is woven and composed of $100 \%$ cotton fibers. Its shedding capacity was determined as $l o w^{1}$.

2. A burgundy pillowcase from the brand Jean-Jacques Benson (JJB); model Satin Deluxe; size $60 \mathrm{~cm} \times 60 \mathrm{~cm}$. This pillowcase is also woven and composed of $100 \%$ cotton fibers. Its shedding capacity is moderate.

These fabrics were chosen in order to present fibers with strong colors easily recognizable in order to simplify the recovery process. It was also necessary to consider colors that would minimize the risk of confusion with fibers unrelated to the simulations (i.e. contamination fibers). Based on a literature search, orange and burgundy fibers appeared to be rarely recovered from the bare skin and hair of living subjects [14,40]. Hence, six orange and six burgundy pillowcases (one per individual and one for preliminary testing, see following section) were bought at Manor supermarkets in Vevey and Geneva (Switzerland) respectively. As new fabrics may have loose surface fibers, pillowcases were machine-washed using a regular cycle $\left(40^{\circ} \mathrm{C}, 60 \mathrm{~min}\right)$ with $100 \mathrm{ml}$ of laundry detergent. Pillowcases of the same color were washed together without any other fabrics. Finally, pillowcases were air dried and stored separately in paper bags.

\subsection{Simulations}

The transfer of fibers was investigated in the context of to two different types of scenarios aimed to represent the prosecution and defense propositions in casework involving potential smothering homicide with a pillow.

\footnotetext{
${ }^{1}$ Shedding capacities were assigned using a cotton scale according to [39] methodology.
} 


\subsubsection{Criminal action - Smothering}

The goal of this simulation set was to represent smothering homicides with pillows. As each criminal case is unique in its circumstances and execution, the decision was made to allow a certain level of freedom in the realization of the criminal smothering simulations. Since there are always unknown and non-reproducible parameters (strength, pressure, contact points, struggling intensity, etc.), it may be difficult to execute the action with the precision of real caseworks. Furthermore, without a total standardization of the activity it is possible to investigate the transfer of fibers in a broad way, which is the aim of this study. Thus, only the duration of the action was imposed and controlled. Considering legal medicine literature, it was decided that smothering simulations for one minute and thirty seconds would be performed, representing a coherent smothering duration that could result in death $[28,41]$.

The greatest challenge when creating the simulations was to find a way to ensure the safety of the volunteers - particularly because we wished to perform intense smothering simulations close to those in real caseworks which may increase the risk of accidents. As a result, it was decided to remove the pillow and perform smothering simulations with only the pillowcase. To assert whether it influenced the transfer of fibers, some simulations were performed for one minute and thirty seconds on a dummy: five smothering simulations with a pillow inside the pillowcase and five with only the pillowcase (Figure 1). This was carried out with the two previously described pillowcases (i.e., orange and burgundy) representing a total of 20 simulations. The face of the dummy was cleaned before each simulation using ethanol. Results showed that the number of fibers transferred was not significantly different between simulations carried out with a pillow inside the pillowcase and those performed without. This conclusion was made for both, orange and burgundy pillowcases. It is important to notice that the results were compared among pillowcases of the same textile and color only (as the shedding capacity of the orange and the burgundy pillowcases is different).
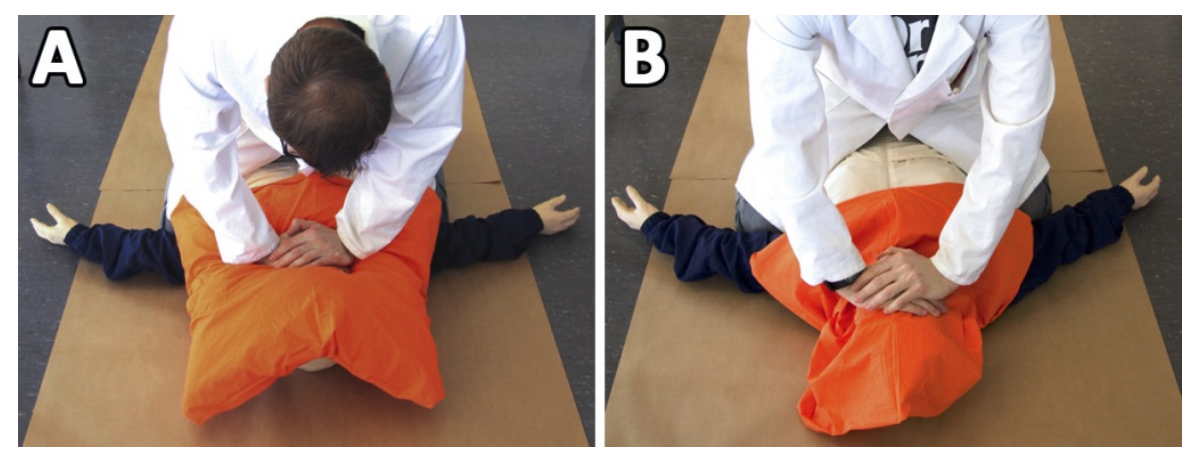

Figure 1. Smothering simulations on a dummy with (A) and without (B) a pillow inside the orange pillowcase (low shedding capacity).

Consequently, each volunteer simulated smothering actions in a lying position for one minute and thirty seconds using only the pillowcases. Simulations were self-inflicted to avoid injuries. Five repetitions were performed, representing ten smothering simulations per individual - five with the orange pillowcase and five with the burgundy one - for a total of 50 criminal activities. To limit contamination, volunteers were requested to wash their face with water before each simulation. As simulations were self-performed, another trained person supervised and documented each conducted experiment (note-taking, photography, etc.).

\subsubsection{Legitimate activity - Night's sleep}

The simulation set representing the legitimate activity that could be claimed by the suspect is represented by one full night's sleep. Thus, each volunteer was asked to sleep one night on each pillowcase after washing their face (volunteer 03 routinely add night cream before going to sleep). Each participant performed five replicates with each pillowcase - five times with the orange pillowcase and five times with the burgundy one - for a total of 50 simulations of legitimate activities. Contrary to smothering simulations, a pillow was introduced within the pillowcases allowing for more 
comfortable, convenient conditions. Experiments were all carried out at the domicile of the participants.

Items were added to the questionnaire in order to investigate some parameters that may have influenced the transfer of fibers during the simulations. The quality (calm or turbulent) and the duration (in hours) of sleep were thus sought. Indication of the head position in the morning was also requested (right, left, back or front side). Sleeping position and duration were not imposed since they could not be controlled.

Finally, precautions were taken to limit the contamination of fibers originating from other bedding fabrics (bed sheet, fitted sheet, other pillowcases...) or nightclothes (pajamas, nightgown...). Therefore, volunteers were asked to remove and/or replace any red, burgundy, purple, orange and even yellow bedding.

\subsection{Recovery, search and data analysis}

Fibers were recovered directly after each experiment with low tack adhesive tapes "Nonperm A2" produced by JAC Serilux (6 $\mathrm{cm} \times 24 \mathrm{~cm})$. A 1:1 taping approach was carried out to preserve the distribution of fibers $[36,42]$.

First, volunteers had to move away from the simulation area in order to avoid contamination. Subsequently, a trained individual applied three adhesive tapes on the face (Figure 2). Eyebrows, nose and mouth position, as well as the outline of the face, were annotated using a permanent ink pen directly on the adhesive tapes. A picture was then taken before tapes were removed and fixed on transparent acetate sheets. Finally, sampling were documented (date, pillowcase, volunteer, activity type, etc.) and sealed in a paper envelope.

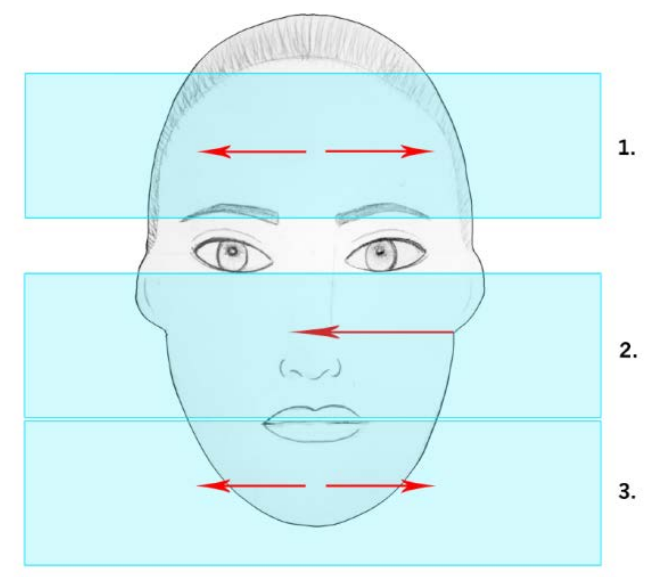

Figure 2. Methodology for fiber recovery using three adhesive tapes. Red arrows indicate the direction of application of tapes.

Tapes were examined under a Leica Wild M3Z stereomicroscope in reflected light, magnification 16x. The search, count and localization of the fibers were performed manually, directly on the tapes. Five zones of $6 \mathrm{~cm} \mathrm{x} 8 \mathrm{~cm}$ were delimited to represent the forehead, left cheek, nose, right cheek and chin (Figure 3). Fibers outside these zones, in the hair region or on the neck were not considered as part of the results. Nevertheless, it is interesting to note that a significant number of fibers was sometimes observed in areas covered by hair (pink areas on the right and left sides of the forehead zone, Figure 3).

Data analysis (statistical computing) and plots were carried out using the open source platform RStudio (Version 0.99.902) and the programming language R (Version 3.3.1). Packages used for data analysis and figure creation were bootstrap, evd, fitdistrplus, fBasics, ggplot2, logspline and reshape2. Interpretation of the results was performed using a Bayesian perspective [9,38]. 

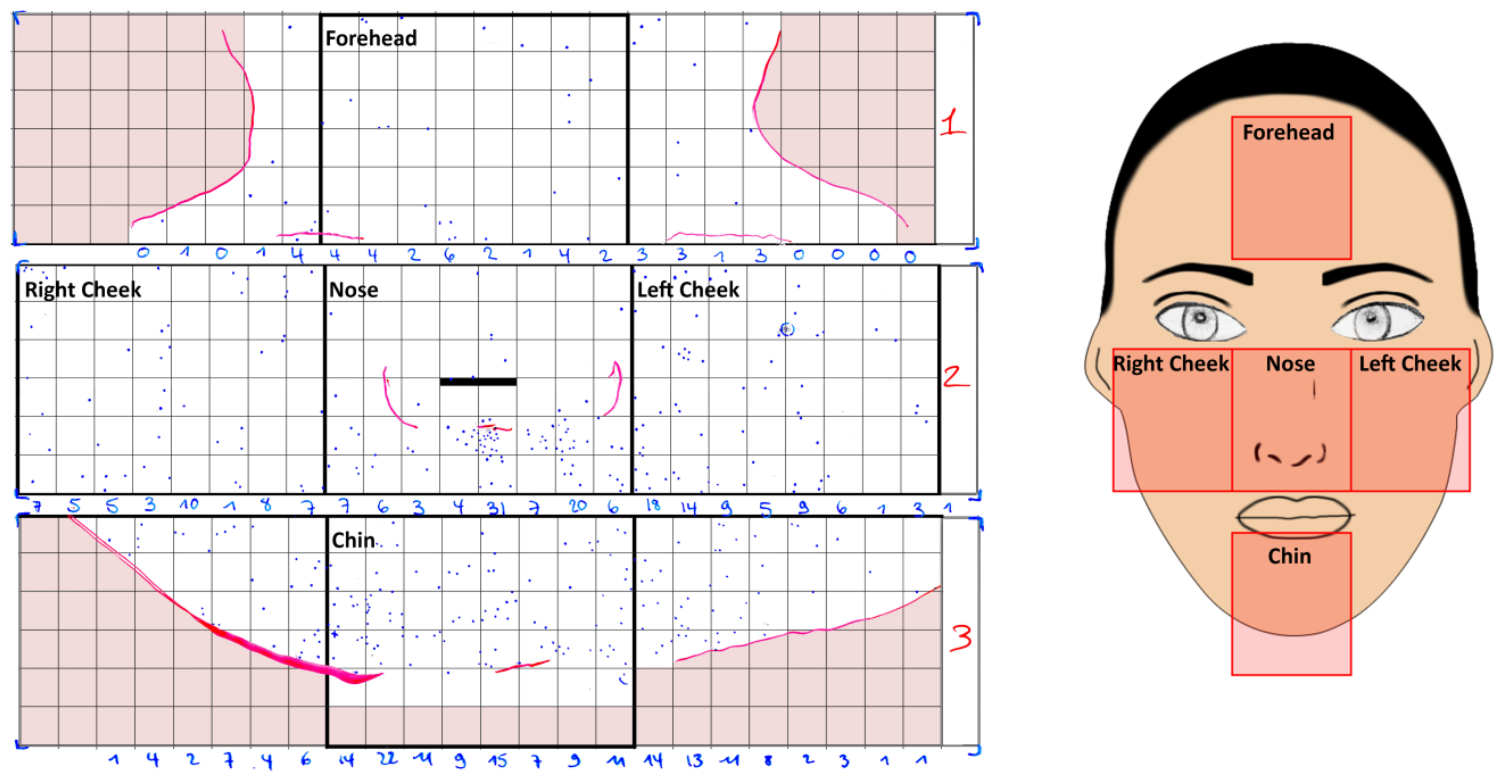

Figure 3. Sampling of a smothering simulation (left) and illustration of the five zones considered in this work (right). Fibers are represented by blue dots on tapes. The outline of the face, eyebrows and nose were also delimitated by red lines. Left and right cheeks are inverted due to a mirror effect.

\section{Results and Discussion}

\subsection{Transfer of fibers}

\subsubsection{Number of fibers recovered and the importance of the shedding capacity}

The number of fibers observed after criminal and legitimate activities, i.e., smothering and night's sleep, respectively, is illustrated in Figure 4 in the form of boxplots. Boxplots were chosen as relevant statistical tools to take advantage of the distribution of the data through their quartiles.

After one night's sleep (legitimate activity), 27 to 154 fibers and 93 and 341 fibers were recovered from the orange and burgundy pillowcases, respectively. After smothering simulations, 57 to 519 fibers, non-differentiable from the orange pillowcase were recovered compared to 88 to 2298 fibers from the burgundy pillowcase. For reasons of readability, three outliers were excluded in Figure 4, corresponding to 2298, 1557 and 1422 fibers recovered after smothering simulations performed by volunteer 01 with the burgundy pillowcase. 


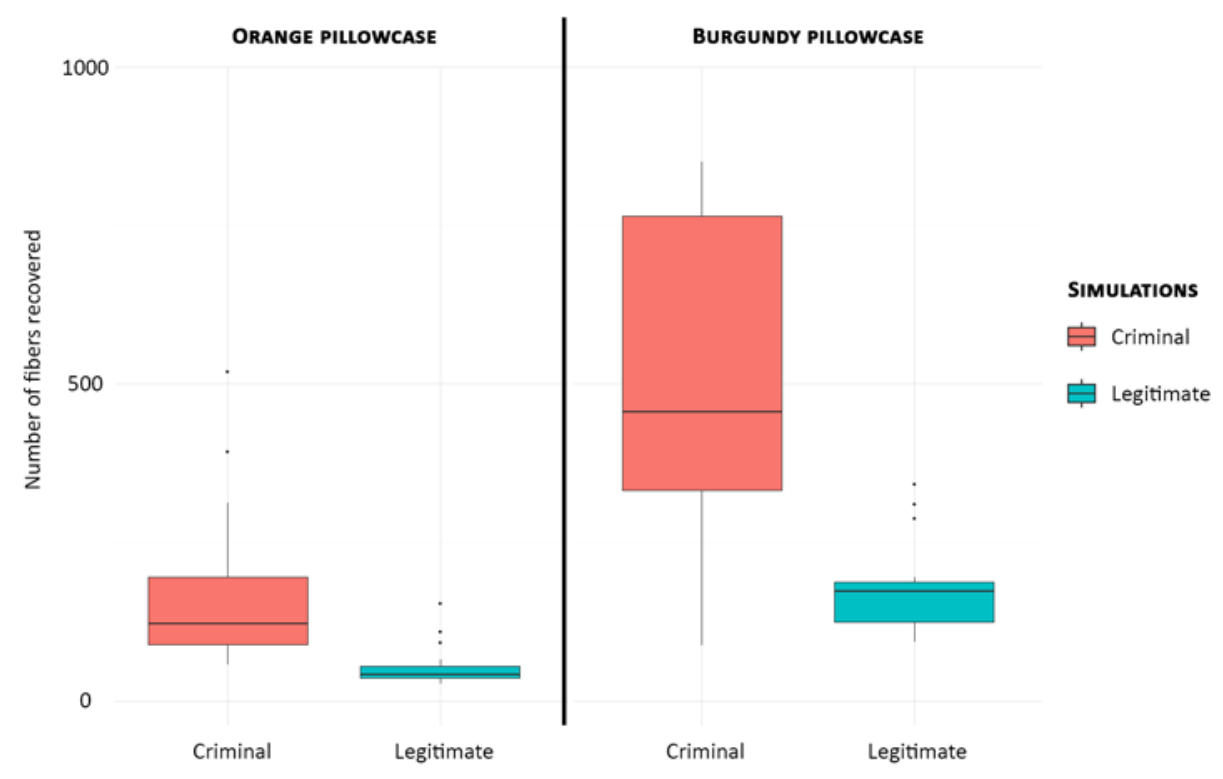

Figure 4. Boxplots of the number of fibers recovered for each type of pillowcases after criminal (red) and legitimate (blue) activities.

When considering the pillowcases separately (orange versus burgundy), usually more fibers were found to be transferred in criminal activities than in legitimate ones. This general trend was also observed when considering participants one by one. This observation supports the literature which states that the intensity of contact significantly influences the transfer of fibers. The more intense the action, the larger the number of transferred fibers $[1,3]$. Thus, more fibers are expected to be present after a smothering than after one night's sleep simulation.

Interestingly, the number of fibers recovered in smothering simulations with the orange pillowcases is relatively close to the number of fibers recovered after one night's sleep on the burgundy pillowcases (Figure 4). This demonstrates that the nature of contact is not the only parameter impacting the number of transferred fibers but that the shedding capacity of the fabrics is also a crucial parameter to be assessed $[43,39]$. To reiterate, the orange pillowcase presents a low shedding capacity compared to the burgundy pillowcase that offers a moderate one - which explains the results obtained. Moreover, it highlights that without any information about the shedding capacity of the fabric(s) it might be impossible to infer on the nature of the activity at the origin of a target fiber group. Therefore, it is not recommended to present any conclusion at the activity level if it is exclusively based on the number of fibers recovered without considering the shedding capacity of the incriminated material.

\subsubsection{Facial distribution of fibers}

Previous case reports showed that the distribution of fibers on the victim's face might be crucial to understanding the activity causing the transfer. For example, the distribution might be useful to reconstruct a potential fabric source or to conclude on a given scenario [32-36,44]. Thus, it was interesting, if not crucial, to examine the distribution of fibers on the face to investigate whether it may help in discriminating between the scenarios presented by this study (smothering or sleep).

The number of fibers recovered in each facial zone for the simulations performed with the orange and burgundy pillowcases are illustrated in Figure 5 and 6, respectively. 


\section{Orange pillowcase}

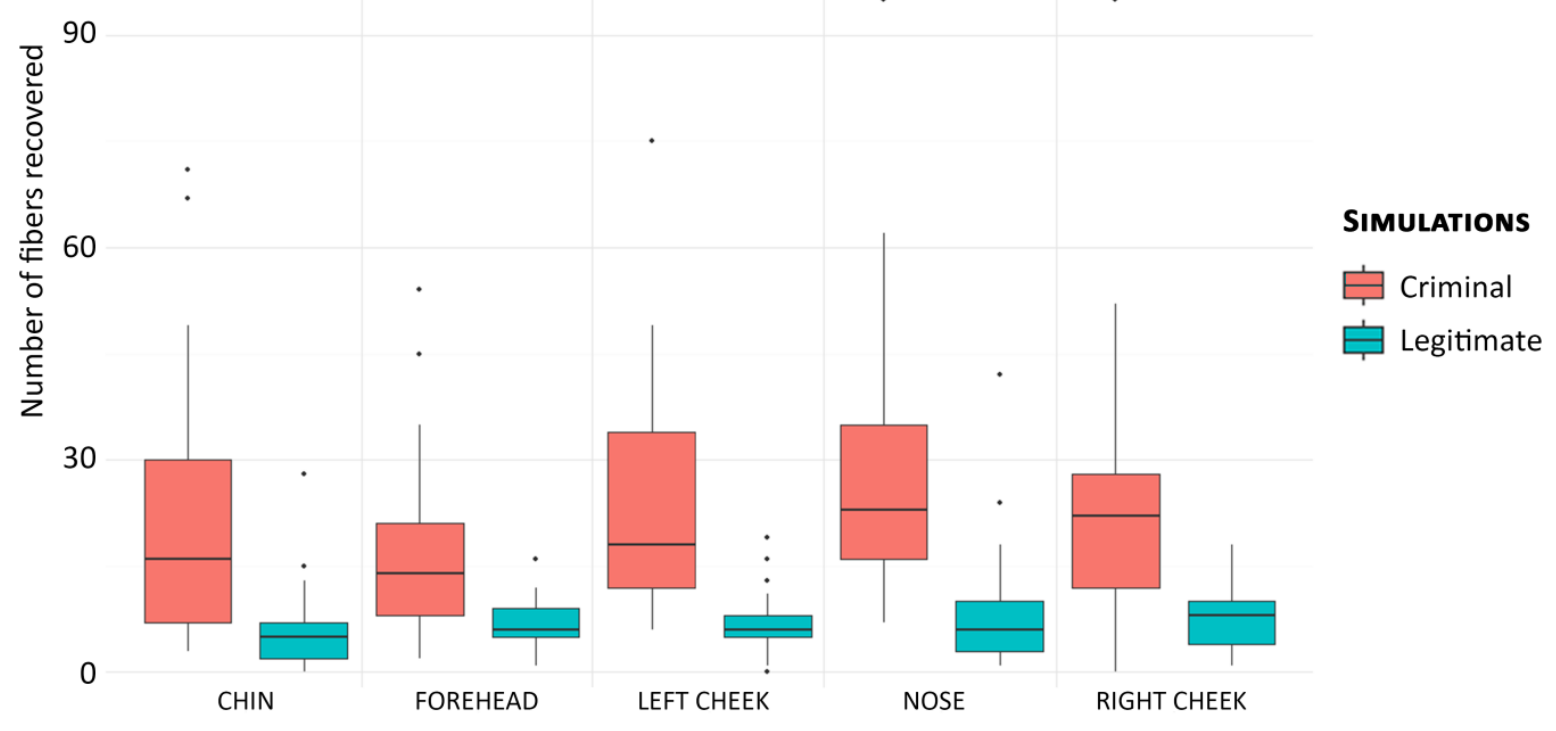

Figure 5. Boxplots of the number of fibers recovered in each facial zone after criminal (red) or legitimate (blue) activities - Orange pillowcase.

\section{BURGUNDY PILLOWCASE}

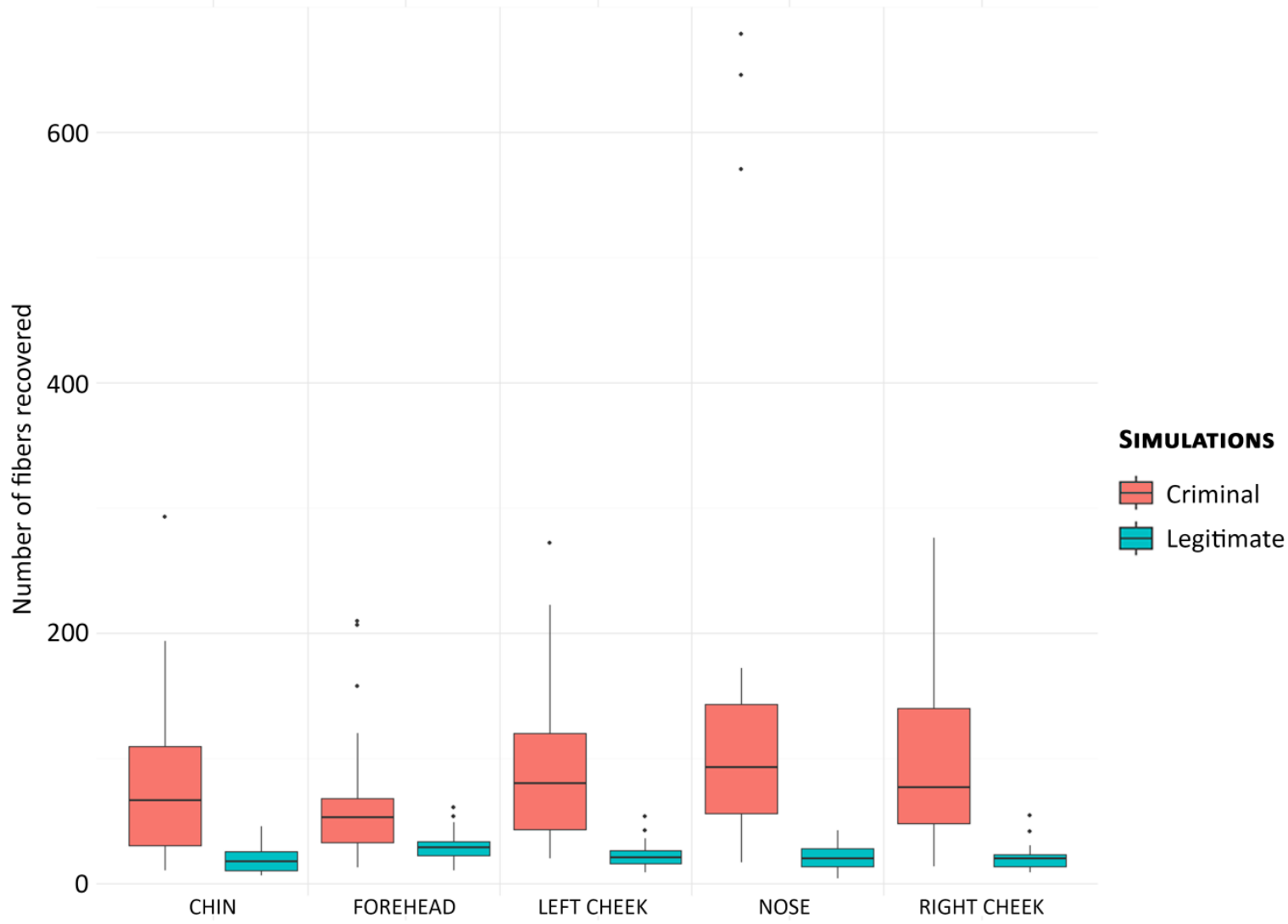

Figure 6. Boxplots of the number of fibers recovered in each facial zone for criminal (red) or legitimate (blue) activities - Burgundy pillowcase.

After criminal simulations there were usually more fibers recovered than after legitimate actions, independent of the zone. This observation further supports the general trend mentioned in the previous 
section. Results also corroborate the influence of shedding capacity of the donor fabric on the transfer, as previously highlighted.

Concerning the distribution of fibers, no significant visual difference was observed for legitimate and criminal simulations performed with neither the orange pillowcases nor burgundy ones. This is something unexpected as more fibers were expected in areas experiencing greater pressure. However, volunteer 01 - a 23 year-old male, clean-shaven with no particular facial care - showed a tendency that differs from other participants. In some replicates of his smothering simulations performed with the burgundy pillowcase (moderate shedding capacity), a higher number of fibers was observed in the nose area than in the others (Figure 7). These observations (obtained from the nose area) are represented by the three outliers previously mentioned (679, 646 and 571 fibers).

\section{BURGUNDY PILLOWCASE - VOLUNTEER 01}

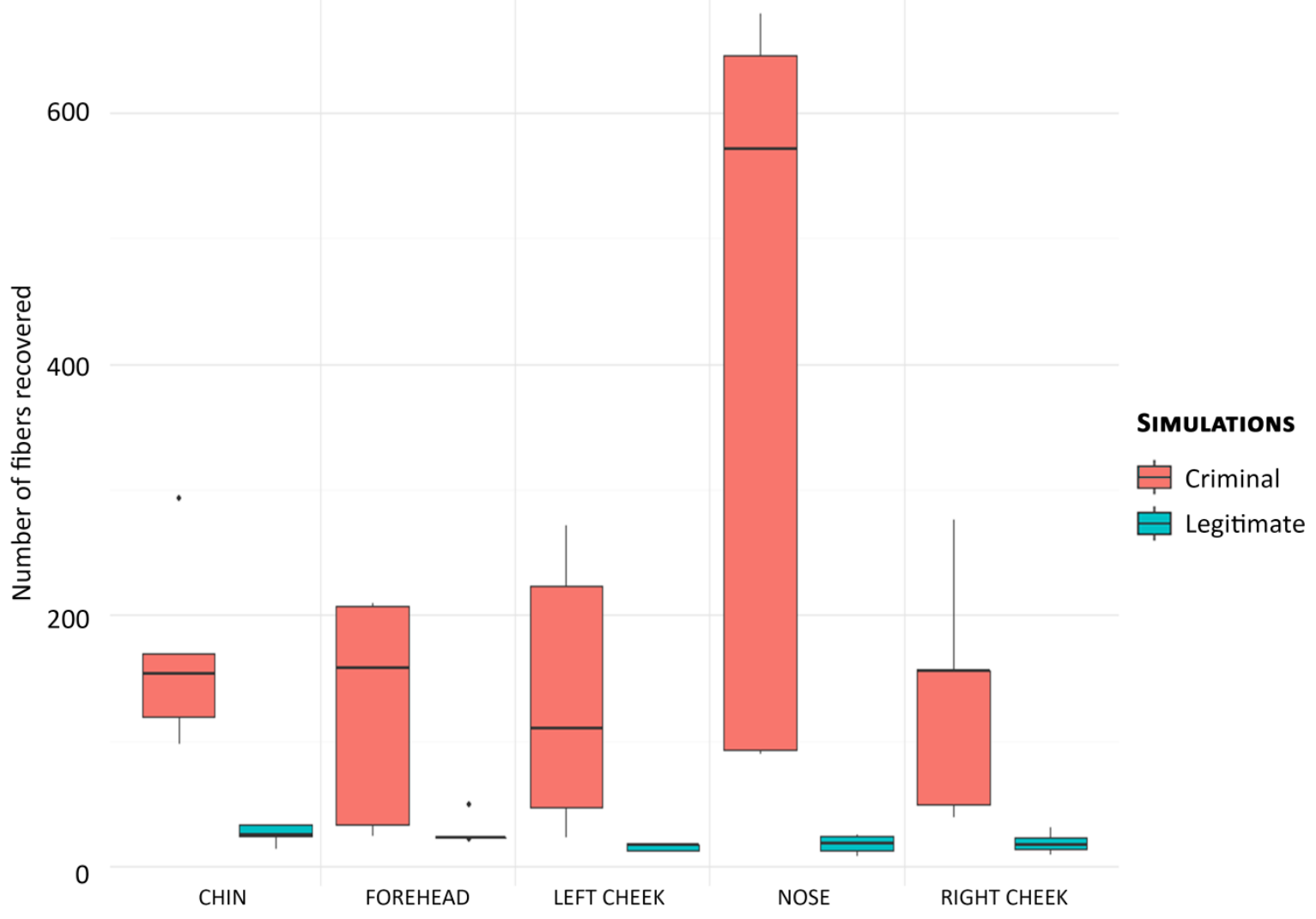

Figure 7. Boxplots of the number of fibers recovered in each facial zone after criminal (red) and legitimate (blue) simulations; Burgundy pillowcase; Volunteer 01.

It was interesting to try explaining the reasons why this phenomenon was observed for replicates carried out by volunteer 01 (replicates one, two and four) and not for all the other simulations and replicates performed by the other participants. Thus, the questionnaires were studied and it appears that two parameters may be the source of these particular distributions. First, the smothering technique might influence the repartition of fibers. As explained in Materials and Methods (section 2), no specific smothering technique was imposed. Documentation showed that two main techniques were used during the simulations (Figure 8): hands were placed either on nose and mouth (Technique 1) or on each side of the cheeks (Technique 2). These techniques might vary in their points of contact and applied pressure. For example, does the first technique generate more pressure on nose whereas the second produces more homogeneous pressure on the whole face? What about the contact surface? Volunteer 01 used both techniques and more fibers were found in the nose area when he was using the first technique whereas volunteers 02 to 05 tended to place their hands mostly on each side of the cheeks (2). Thus, depending on the modus operandi, the distribution of fibers might vary and be 
interesting to investigate these points in more detail and should be considered in future simulationbased studies.
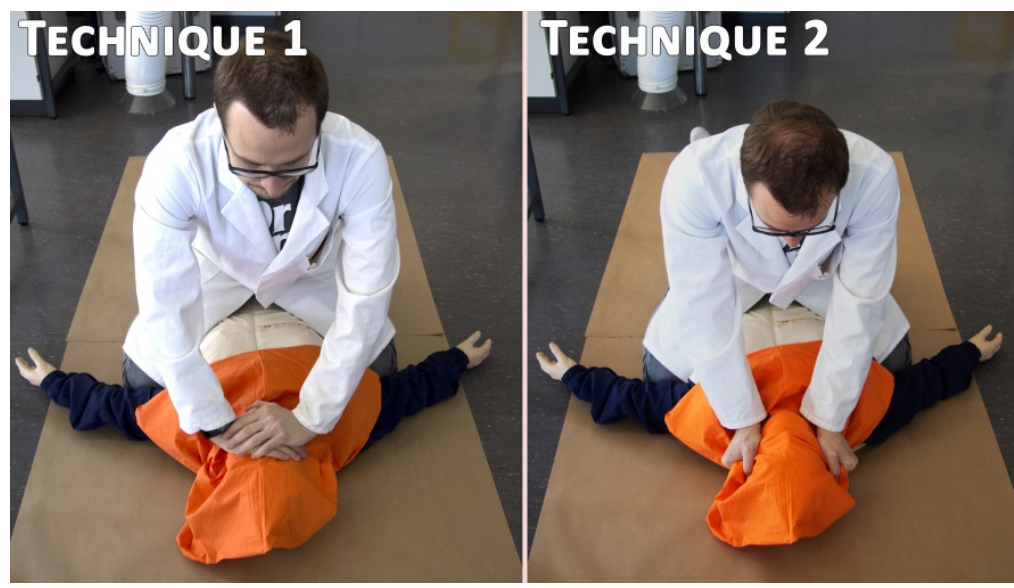

Figure 8. Illustrations of the two techniques used by the participants in smothering simulations. Hands are placed on the nose and mouth (Technique 1) or on each side of the cheeks (Technique 2).

The amount and intensity of the movement - or friction - might also influence the number of fibers transferred. Few years ago, literature mentioned that without any friction (static contacts), only little transfer occurs. [18]. Generally, limited movements are produced during the sleep (legitimate activity), which might explain the low number of fibers recovered (associated with the shedding capacity and pressure, as previously discussed).

In smothering simulations, volunteer 01 applied intense pressure and struggle, involving a lot of friction. However, it also appeared that a significant number of smothering simulations involved limited movements where some volunteers remained relatively inert, focusing mainly on pressure less than movement. Volunteer 02 simulated particularly light pressure and little movement in her simulations. In these simulations, fewer fibers were recovered than from those involving high struggling movements.

Which situation represents reality? Which technique is most likely encountered in real cases? No answer can be provided as it depends on the victim, the perpetrator and lastly, the circumstances of the case. Was the victim in good health or was she debilitated? Was she able to struggle and if so, to what degree? Nevertheless, results showed that the quantity of movement and the modus operandi significantly impact the distribution and the number of transferred fibers. Thus, the distribution of fibers should systematically be considered in homicide cases as it might be highly relevant for the investigation.

It should also be noted that based on present data and the lack of struggling involved in certain simulations, the number of fibers transferred in real cases may, in fact, be underestimated. If investigations suppose/assume intense struggling between the victim and their assailant, a higher transfer of fibers is expected. On the other hand, data could also represent some real case circumstances as smothering homicides involved mostly debilitated, disabled, very young or very old victims that are unable to defend themselves against their assailant (with high resistance) [28-30]. Thus, the health condition of the victim has to be considered in the evidence interpretation process since it could significantly affect the transfer of fibers. Results also highlighted that it might be difficult to provide information about the activity at the origin of the recovery in cases involving small amount of target fibers.

The data provided by this study allows for a better understanding of the transfer of fibers and several of the key influencing parameters involved. It also highlights that interpretation at the activity level can be carried out using fibre evidence. It must be emphasized, however, that the present data cannot 
be used directly as a substitute for the interpretation of real caseworks. Indeed, in real investigation processes, it is crucial to carry out some simulations using appropriated case circumstances and incriminated textile materials.

\subsubsection{Influence of the other parameters}

As previously pointed out, the number of transferred fibers and their distribution might be influenced by the activity itself - mainly the pressure and the movement (friction) applied on the face - and by the shedding capacity of the pillowcases (donor fabrics). No other influencing parameter was highlighted in this work. Neither facial hair nor cosmetics (cream, make-up remover, etc.) nor gender affected the transfer of fibers in both scenarios (criminal and legitimate activities). Similarly, the repartition and number of transferred fibers during the night (legitimate activity) were not impacted by the head position in awakening nor the duration and quality of sleep. The quality of sleep - which was self-assessed in the questionnaire (categories: calm, turbulent or unknown) -also had no influence on the transfer of fibers.

This might be due to the small number of participants since only five individuals were performing the simulations. Despite this experimental restriction, a total of one hundred simulations were conducted fifty smothering actions and fifty nights' sleep per participant - which is a considerable number. This also represents fairly realistic investigation conditions since it is uncommon to perform so many simulations to assess fiber evidences. Nevertheless, it is still necessary to vary specifically those characteristics and lifestyle habits (e.g. facial cosmetics, facial hair, position of sleep, etc.) in future studies to investigate their potential impact not only on fiber transfer, but also their persistence.

\subsection{Bayesian interpretation of the results}

When a group of target fibers indistinguishable from a suspect's fabric is found on the victim, it is crucial to assess the evidentiary value of the findings. In this research a Bayesian decision perspective is used for interpreting the data. Readers unfamiliar with Bayesian interpretation and likelihood ratios (LR) are encouraged to consult the literature $[9,37,38]$.

Results of this study showed that the main difference between the transfer of fibers in smothering and night's sleep scenarios lies in the number of fibers recovered. Unfortunately, the general distribution of fibers was relatively homogeneous except for some simulations carried out by volunteer 01 . Thus, in the present study, interpretation will only be based on the number of transferred fibers in either of the scenarios without considering their localization. If specific areas could have been highlighted after simulations (e.g. nose and forehead), only fibers present in the relevant areas would have be considered for the interpretation process.

\subsubsection{Determination of propositions and development of likelihood ratio}

To simplify the complexity of the following sections, let us refer back to the case presented in the introduction. After recovering 170 orange cotton target fibers from the victim's face, prosecution claims that the suspect smothered the victim with her pillow covered by a $100 \%$ orange cotton pillowcase. The recovered target fibers were not differentiable from the fibers originating from the pillowcase. According to the suspect's allegations, however, the victim died during her sleep. Thus, the following set of propositions can be used:

Proposition 1: The victim was smothered with the pillow

Proposition 2: The victim slept on the pillow and died naturally or accidentally respectively

This set of propositions implies an interpretation at the activity level. As mentioned in the introduction, interpreting the observations at the source level would be uninformative as the comparison material is the same under both propositions, represented by the pillow of the victim. This would result in a LR equal to 1 , meaning that the probability to observe the target fibers whether 
proposition 1 or proposition 2 is true is equivalent. Thus, the evidence is unable to provide the investigation with any conclusive information.

The general form of the likelihood ratio for fiber evidence at the activity level is the following [37]:

$$
L R=\frac{t_{n} b_{0}+t_{0} b_{1, n} \gamma}{t_{n}^{\prime} b_{0} \gamma+t_{0}^{\prime} b_{1, n} \gamma}
$$

where $t_{n}$ and $t_{0}$ represent the transfer of ' $\mathrm{n}$ ' or zero fiber during the activity considered under proposition 1 (smothering), $t_{n}^{\prime}$ and $t_{0}^{\prime}$ the transfer of ' $\mathrm{n}$ ' or zero fiber during the activity considered under proposition 2 (night's sleep), $b_{1, n}$ and $b_{0}$ the presence of ' $\mathrm{n}$ ' or zero fiber from the background and $\gamma$ the occurrence of the recovered fibers (orange cotton).

As previously stated, the fabric considered under proposition 2 is the same as under proposition 1 in our case scenario meaning that they both involve the pillow of the victim and its orange cotton pillowcase. Thus, it is no longer relevant to consider the occurrence of the fibers in " $t_{n}^{\prime} b_{0} \gamma$ ”, therefore the likelihood ratio was adjusted as follows:

$$
L R=\frac{t_{n} b_{0}+t_{0} b_{1, n} \gamma}{t_{n}^{\prime} b_{0}+t_{0}^{\prime} b_{1, n} \gamma}
$$

In the case example, 170 fibers were recovered from the victim's face. According to literature, it is highly uncommon to observe more than a few fibers coming from the background, independently of the substrate [20,45-49]. The largest group of orange cotton fibers found randomly was 33 fibers in a study by Grieve et al.[47]. It is interesting to note that only the color and type of the fibers were considered in this study and that the fibers were recovered on canteen seats. Since our case example involves a large group of fibers (170 units), it can be assigned that $b_{1, n}$ and $b_{0}$ are closed to 0 and 1 , respectively, and thereby can be neglected. It is important to note that these simplifications must be discussed in situations where only few fibers are recovered on the victim or in cases where the victim has had frequent non crime-related contact and activities/interactions involving textile fibers (e.g. working in the automotive industry or as a fashion designer, etc.).

Consequently, the likelihood ratio for our case example is:

$$
L R=\frac{t_{n}}{t_{n}^{\prime}}
$$

with ' $n$ ' being the number of fibers recovered. In our case example, $n=170$.

Hence, only the probabilities related to the transfer remain, showing the importance to be able to investigate and discuss the transfer of fibers at the activity level considered by the propositions. On the one hand, what is the probability of observing 170 fibers when they were transferred during the smothering of the victim with the pillow (proposition 1)? On the other hand, what is the probability to recover those fibers from the face of the victim after sleeping on the pillow (proposition 2)?

\subsubsection{Interpretation without any specific data}

Without any data pertaining to the transfer of fibers in both scenarios, it might be difficult to assign those probabilities. Practitioners should refer to their own experience or to the literature in order to assess the evidentiary value of the evidence.

To our knowledge, however, no specific data regarding the number of fibers recovered on the face of victim's in smothering homicide cases (criminal activity) or after a night's sleep (legitimate activity) have existed prior to this work. Nonetheless, it is possible to refer to studies that have investigated the transfer of fibers on the skin of living subjects [14] and even on pig skin [50] in order to extrapolate the potential results of smothering scenarios. For nights' sleep, it is more difficult as it is uncommon to 
find data about the transfer of fibers in activities involving soft and extended contacts. Studies devoted to the transfer of fibers on car seats might be the most appropriate information sources thus far $[18,43]$.

Considering these data and the estimation of the shedding capacity of the incriminated pillowcase, it is possible to assess a LR based on literature values. However, since the scenarios found in literature are quite different from the situations considered in propositions 1 and 2, the scientist would have to incorporate uncertainty, which might ultimately lead to inconclusive LRs.

It is therefore recommended to carry out simulations for real case investigations with conditions close to those of the case. Ideally, the simulations will be performed with the evidence material or another fabric resembling the shedding capacity - another pillowcase in our scenario - since the shedding capacity is crucial for the interpretation process. This approach is the most way to produce relevant and useful results.

The results provided by this study could also be considered, however, one would have to assume that the shedding capacity and composition of the pillowcase involved in the real case are similar to the one of the pillowcases used in this study (e.g. by using shedding scales [39]). Moreover, data obtained in this survey should be adjusted to the case circumstances in relation to the dimension, composition and shedding capacity of the pillowcase but also to how events could have unfolded (amount of friction, technique, etc.). Due to difficulties in managing adequate adjustments, it is highly recommended to systemically perform case oriented simulations in order to complete the data found in the literature.

\subsubsection{Investigation of data and calculation of the likelihood ratio (LR)}

\subsubsection{Orange cotton pillowcase}

\section{Density curves}

Considering the results obtained for the orange pillowcase, it appeared that the data fit a log normal distribution both, for the criminal activity (smothering, proposition 1) and legitimate activity (night's sleep, proposition 2) represented by the probabilities previously discussed, $t_{n}$ and $t_{n}^{\prime}$, respectively (Figure 9). 


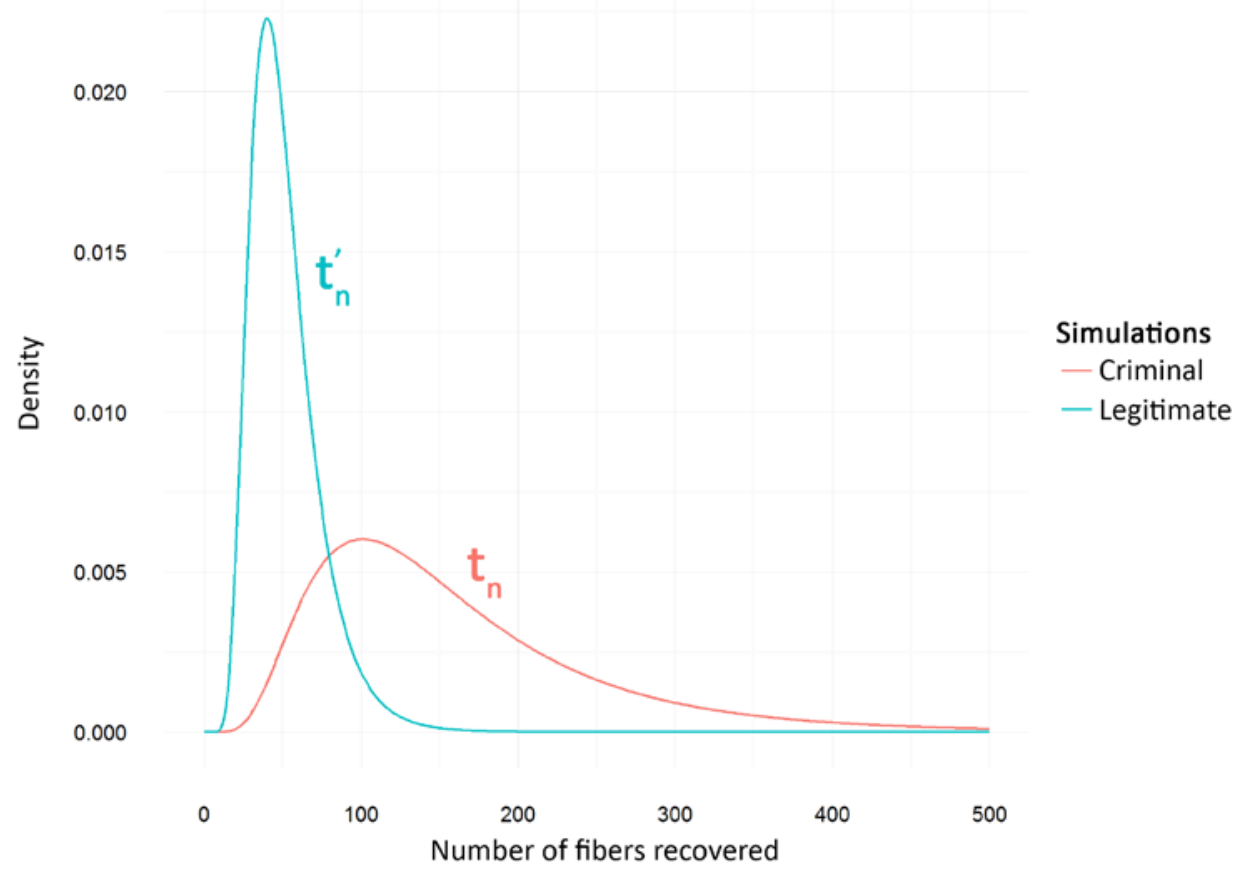

Figure 9. Density curves of the number of orange cotton fibers (orange pillowcase, low shedding) in criminal (red line) and legitimate (blue line) scenarios.

As seen in Figure 9, there is a significant overlap of the two distributions. Considering the whiskers of the boxplots showed in Figure 4, this overlap was expected. It is also interesting to note that log normal distributions could underestimate the probability to encounter a large amount of fibers. Indeed, the distributions are asymmetric and present heavy right tails, namely for criminal activity $\left(t_{n}\right.$, red line). Thus, instead of fitting a log normal distribution, it could be interesting to consider extreme value analysis (EVA) for the right tail of the distributions [51,52]. EVA is a branch of statistics allowing the estimation of uncommon events that have not been observed previously (i.e., after the simulations in this case). Thus, it could avoid underestimating the probability to observe an important number of fibers, and consequently avoid overestimation of the LR. However, this type of analysis is beyond the scope of this article which intends to remain at a moderate level of data analysis. Nevertheless, if the number of target fibers recovered is far from the median and considered as an extreme event, EVA should be conducted.

An important fact to remember is that only 25 simulations were performed for each scenario. As previously discussed, this is not a compelling number but it is actually, fairly representative of what might be expected in real case investigations. Performing too many simulations would lead to a loss of time and money (data acquisition and analysis), a pragmatic limitation that practitioners are required to deal with.

What is the impact of such a limited dataset? What is the impact on the distributions if one simulation differs by 50 fibers? To investigate the robustness of the model, a bootstrap of the data was carried out using R software. Concretely, this process follows a classical urn problem. All 25 simulations were placed into a fictional urn and one of them was drawn. The number of fibers associated with the simulation was recorded and then put back into the urn. A total of 25 draws were made until depicting a new dataset. A total of a thousand replicates were performed representing a $1000 \times 25$ matrix for each proposition (smothering and night's sleep). The two log-normal parameters (location and scale) were then calculated for each replicate. Therefore, 1000 distributions for criminal activity (smothering) and 1000 other distributions for legitimate activity (night's sleep) were determined. These are represented in Figure 10 in the form of boxplots. Concretely, for a given number of fibers recovered on the victim's face ( $x$ axis), the probability of observing this number of fibers is 
represented with a boxplot (density, y axis). In this way, density curves including uncertainty can be represented for t (proposition 1, smothering, red boxplots) and t' (proposition 2, night's sleep, blue boxplots). Due to readability concerns some whiskers were truncated, outliers are not shown and the number of fibers considered was limited to 300 .

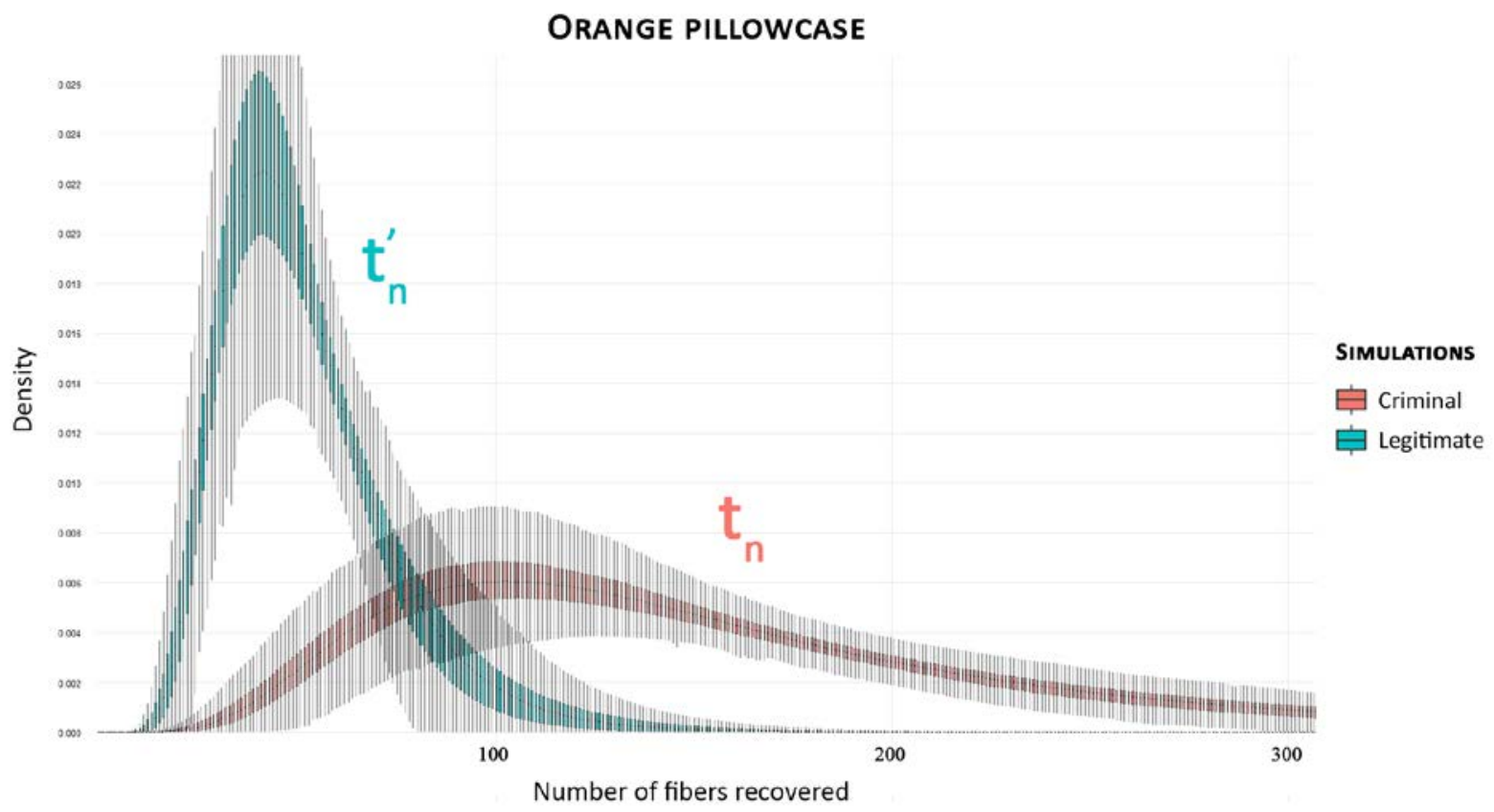

Figure 10. Boxplots representing the number of orange cotton fibers (orange pillowcase, low shedding) recovered in criminal (red) and legitimate (blue) activities after bootstrapping the data (1000 replicates).

The dispersion of data shows that the model is not very robust. Due to the number of simulations ( $\mathrm{n}=$ 25 for each scenario) this was expected. Mainly, this highlights that the results should be interpreted with caution and that the conclusions should be moderate.

\section{Likelihood ratio (LR)}

As previously developed, the LR to consider in a case scenario involving a large group of target fibers is:

$$
L R=\frac{t_{n}}{t_{n}^{\prime}}
$$

To determine the likelihood ratio, a ratio of the two density curves illustrated in Figure 10 should be calculated rather easily, given that they represent the expected number of fibers after smothering simulations (proposition 1, red boxplots) and after a night's sleep simulations (proposition 2, blue boxplots). The distribution of $\log _{10}(\mathrm{LR})$ is illustrated in Figure 11 in form of boxplots. As before, this allows for illustrating the uncertainty and appreciating the LR repartition. For readability concerns, the number of fibers considered was limited to 300 fibers and boxplot outliers were removed. 


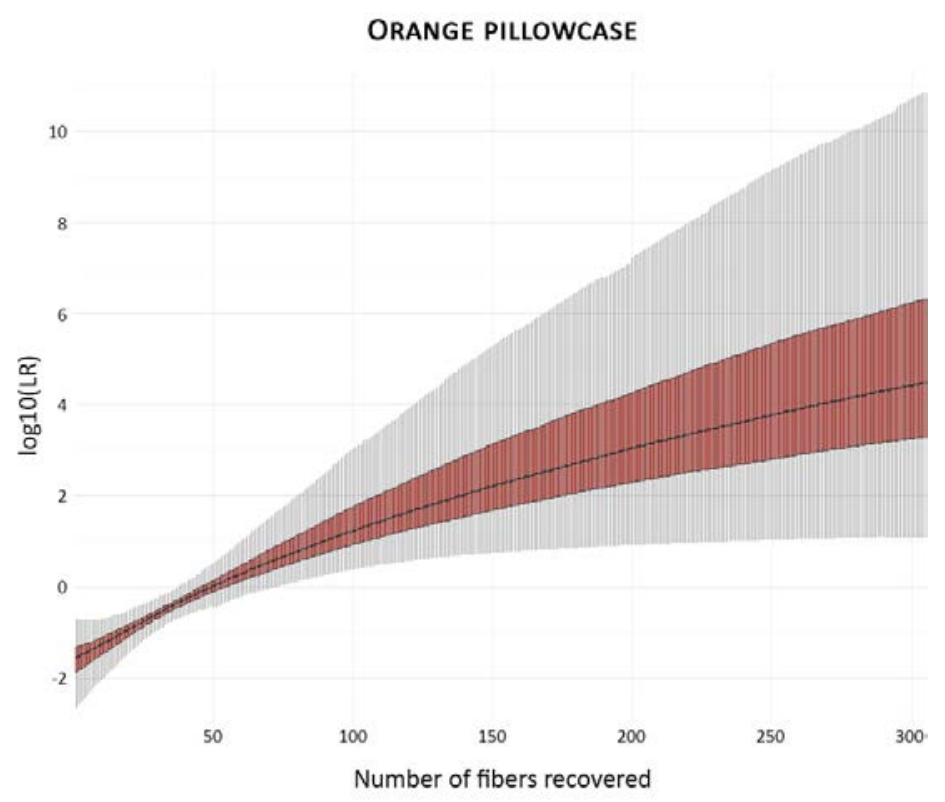

Figure 11. Boxplots representing the distribution of $\log _{10}(\mathrm{LR})$ calculated for the orange cotton pillowcase (low shedding) after bootstrapping the data (1000 replicates).

The further the number of fibres is from the observations made during simulations, the larger the uncertainty on the LR. This is a consequence of the low number of simulations performed and highlights how unusual it was to observe large groups of fibers in this case. Indeed, the number of fibers observed in the simulations performed with the orange pillowcase was comprised mostly between 50 and 200 fibers (Figure 4). Thus, with a high number of fibers, it is crucial to discuss and moderate the conclusions. To this end, EVA should be conducted on extreme and unexpected target fiber numbers (as previously mentioned). Since the data repartition presents a heavy right tail, it might be interesting to model the extreme values with a Fréchet distribution [52].

\section{Case example}

To demonstrate what kind of information can be extracted from the LRs distribution (Figure 11), we return to our case example where 170 orange cotton fibers were recovered on the face of the victim. For the purpose of illustration, let us assume that the pillowcase collected in the example presents a low shedding capacity similar to that of the orange pillowcases used in this work. The likelihood ratio is:

$$
L R=\frac{t_{170}}{t_{170}^{\prime}}
$$

To assess the probative value of the observations (170 fibers), we must refer to the density curves of the orange pillowcase (Figure 10) but more specifically, to their associated LRs distribution (Figure 11). To calculate the likelihood ratio, we simply have to refer to the median of the distribution and record the value - precisely, the $\log _{10}(\mathrm{LR})$ - corresponding to a recovery of 170 fibers (Figure 11 ). The use of the median appears to be a good compromise in this situation since this statistical value is less skewed by extreme values than the mean. According to the results of this survey, it is common to deal with a group of 170 fibers in smothering scenarios with this type of pillowcase. In other words, the size of the evidence fiber group lies within the interval covered by this article. Thus, an extreme value analysis of the data should not be required for the assessment of the evidentiary value of such a group of target fibers. This would not have been the case if the number of fibers recovered were outside the boxplots whiskers.

According to Figure 11, for 170 target fibers recovered, $\log _{10}(\mathrm{LR})$ is equal to 2.15 . Hence, the value of the likelihood ratio for the case example is approximately 140 (since $10^{2.15}=141.25$ ). This means that 
it is 140 times more likely to observe such a group of target fibers if the victim was smothered with the pillow rather than if the victim slept on the pillow and died naturally (or accidentally). Using a verbal scale, the results support the proposition that the victim was smothered with the pillow more so than the proposition whereby the victim slept one the pillow and died naturally or accidentally. This support is qualified as strong [53].

\subsubsection{Burgundy cotton pillowcase}

\section{Density curves}

Similar investigation of the results was carried out on the number of fibers recovered in simulations performed with the burgundy pillowcase exhibiting a moderate shedding capacity. Considering the number of burgundy cotton fibers, the data could also be fit with log normal distributions in criminal (smothering, proposition 1) and legitimate activities (night's sleep, proposition 2). As with the orange pillowcase, the respective distributions are represented by the probabilities $t_{n}$ and $t_{n}^{\prime}$ of the LR at the activity level (Figure 9).

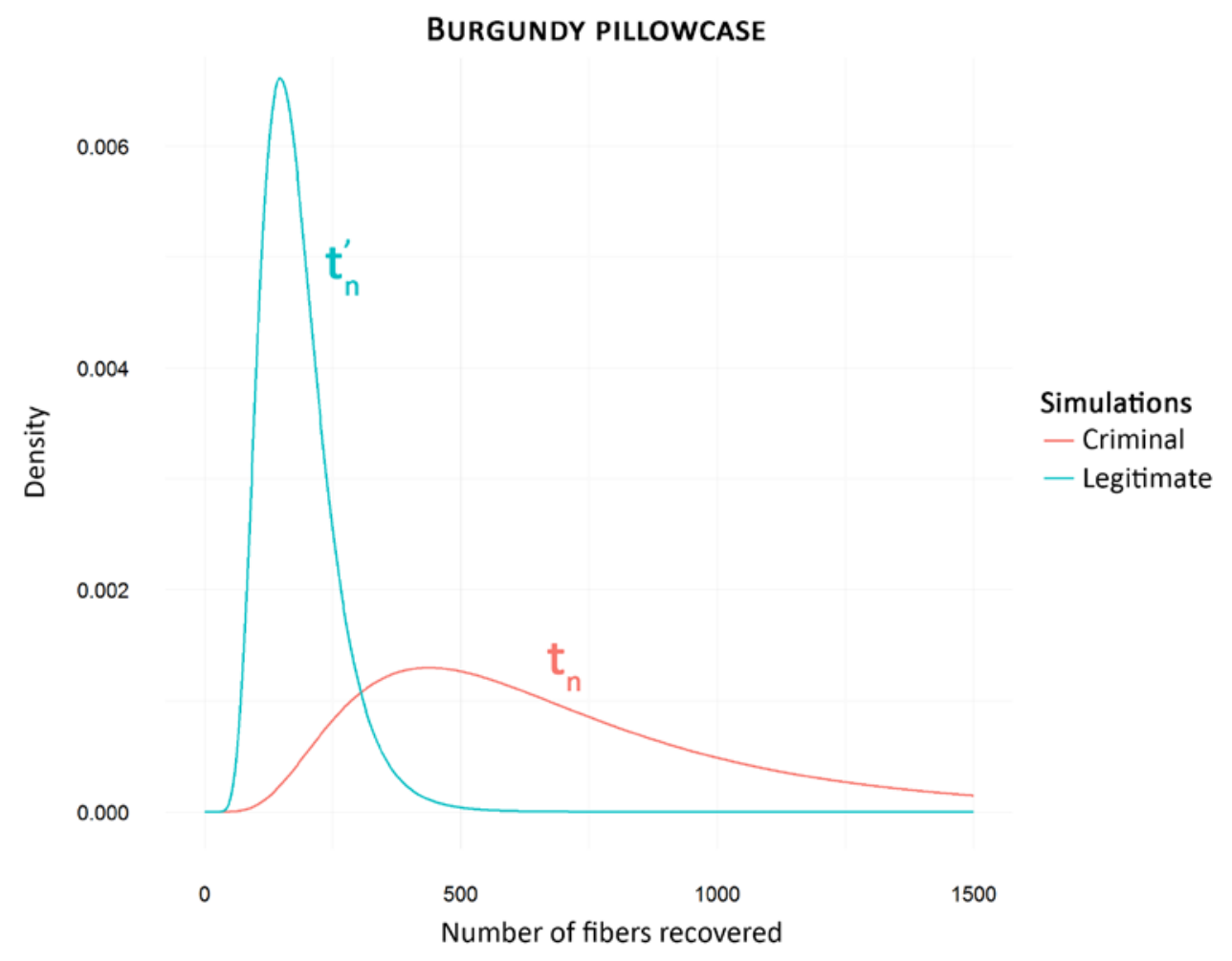

Figure 12. Density curves of the number of burgundy cotton fibers recovered (burgundy pillowcase, moderate shedding) in criminal (red line) and legitimate (blue line) activities.

A significant but again expected overlapping of the two distributions was observed. This representation simply corroborates the observations made with the boxplots (Figure 4). Considering that the number of simulations performed was similar as for the orange pillowcase, it was also decided to investigate the data using the bootstrap approach. One thousand replicates of 25 random draws with replacement were carried out automatically using $\mathrm{R}$ software. The results were represented by a large $1000 \times 25$ matrix for each proposition (smothering and night's sleep activities). The location and scale (parameters of the log-normal distribution) were then determined and the associated distribution was built. These distributions are illustrated in Figure 13 in form of boxplots (facilitating the representation of the results). Due to readability issues, some whiskers were cut, outliers are not shown and the number of fibers illustrated was limited to 800 . 


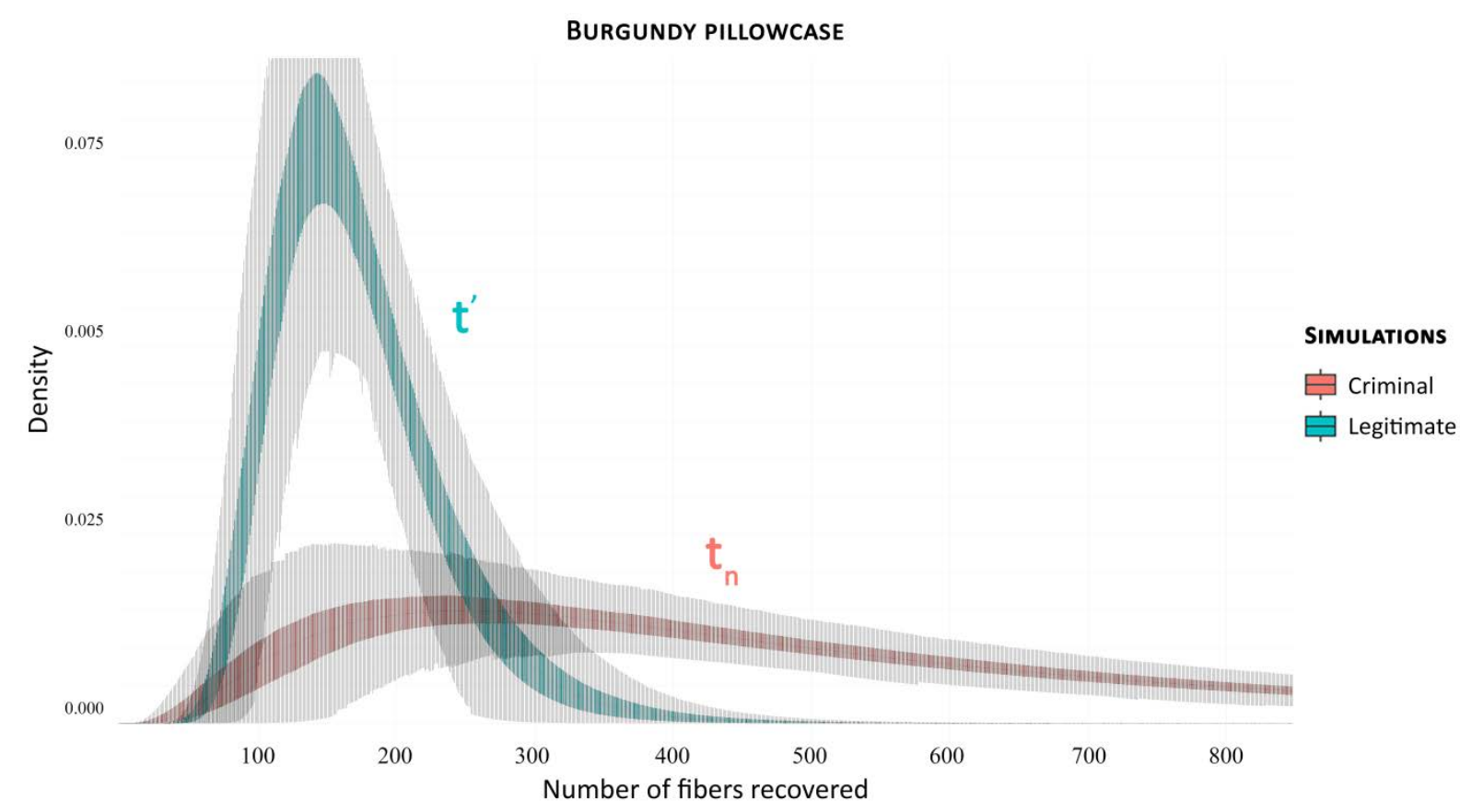

Figure 13. Boxplots illustrating the number of burgundy cotton fibers (burgundy pillowcase, moderate shedding) recovered in criminal (red) and legitimate (blue) activities after bootstrapping the data (1000 replicates).

As for the orange pillowcase, the dispersion of the data clearly illustrates the weakness of the model. The model is even less robust than the one created for the orange pillowcase (Figure 10) as the overlap was increased by bootstrapping the data. Again, this is a direct consequence of the limited, but realistic, number of simulations carried out ( $\mathrm{n}=25$ per scenario). It shows that if one result changed, the impact on the model might be significant. Thus, as previously highlighted, precautions should be taken when assessing the probative value of the fiber evidence.

\section{Likelihood ratio (LR)}

As for the orange pillowcase, to calculate the likelihood ratio for burgundy pillowcase (moderate shedding capacity), the densities curves should simply be divided (Figure 13). Mainly, the probability (i.e., density)of observing a given number of fibers in smothering simulations ( $t_{n}$, proposition 1 ) has to be divided by the probability of recovering the same number of fibers after night's sleep simulations $\left(t_{n}^{\prime}\right.$, proposition 2). The resulting $\log _{10}(\mathrm{LR})$ distribution is illustrated in Figure 14 in form of boxplots. Only the distribution between 0 and 800 fibers is shown and outliers were removed for the improvement of readability. 


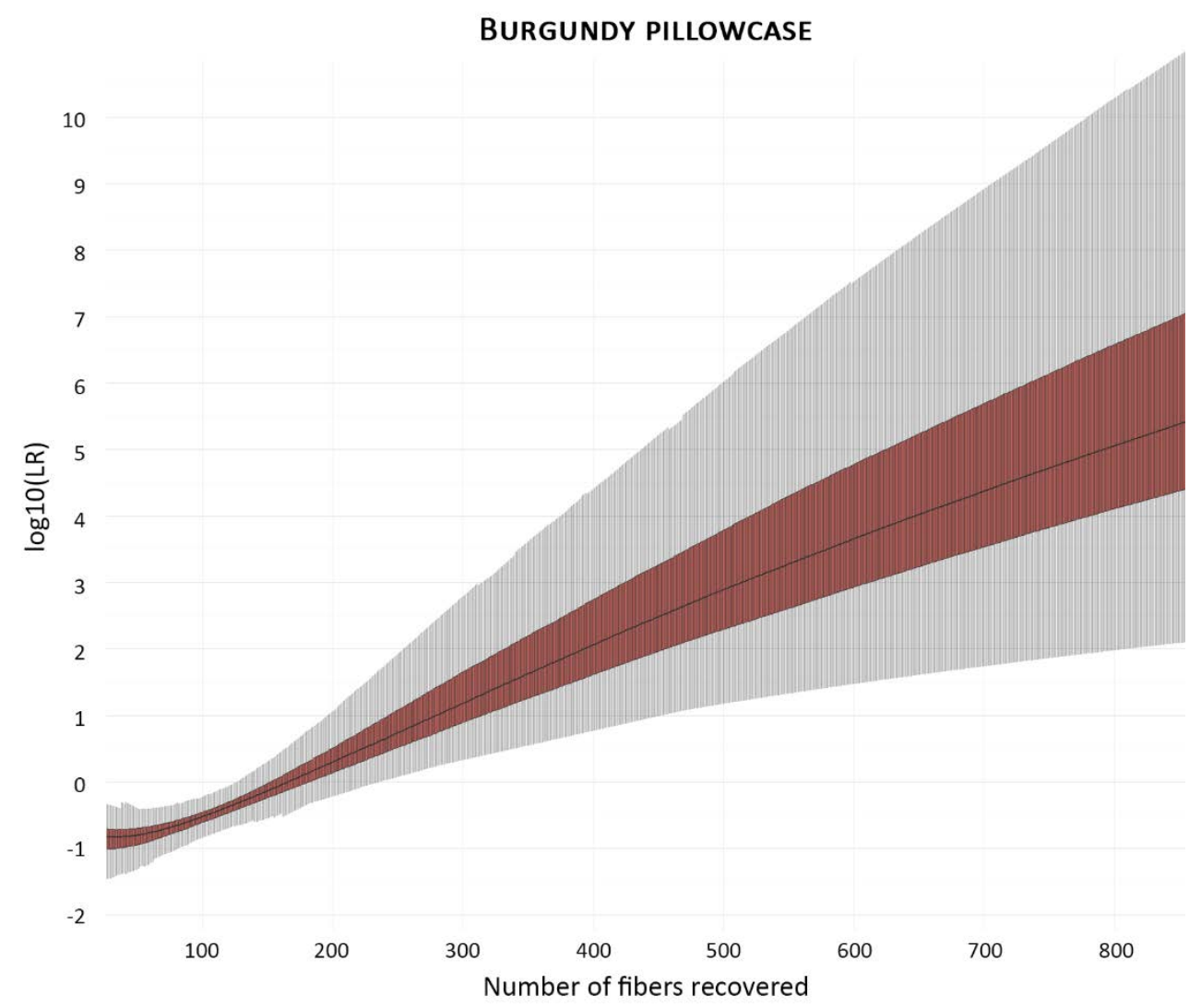

Figure 14. Boxplots representing the distribution of $\log _{10}(\mathrm{LR})$ calculated for the burgundy cotton pillowcase (moderate shedding capacity) after bootstrapping the data (1000 replicates).

The distribution of the likelihood ratios is comparable to the one obtained for the orange pillowcase (Figure 11). The main difference lies in the number of fibers recovered (abscise) - presumably due to differences in shedding capacity - but the general shape remains similar. It was also observed that the higher the number of fibers recovered from the interval covered by the article, the higher the uncertainty. Once again, for unexpected observations - or uncommon recoveries - an extreme value analysis should be considered to specifically parameterize the tails of the data distributions and moderate the increase in likelihood ratios. This would avoid irrelevant, extremely high support for either proposition.

\section{Conclusion}

The research conducted in this study brings forth new knowledge about the transfer and distribution of pillowcase fibers onto the face of an individual involved in smothering homicide as well as in a legitimate night's sleep activities. This information will be useful for forensic fiber specialists, notably for investigations where the central challenge is to determine whether the evidence was transferred as a result of a criminal action or through regular activity (i.e., sleep). Thorough knowledge of the transfer of fibers in each incriminate action (i.e., prosecution and defense proposition) is then required to propose relevant interpretation at the activity level.

Results showed that the shedding capacity of the donor fabric (pillowcase) was the greatest influencing parameter affecting the number of transferred fibers. The nature of the contact seemed also to impact the transfer of fibers, notably the modus operandi and the amount of friction. Further research should be conducted to investigate these parameters.

For a given type of activity, more fibers were recovered in experiments carried out with the burgundy pillowcase (moderate shedding capacity) than those performed with the orange one (low shedding capacity). It is very interesting to point out that there was a similar number of fibers recovered during 
the legitimate scenario (night's sleep) performed with the burgundy pillowcase and during the criminal simulations (smothering) using the orange pillowcase. This information proves/emphasizes the need to consider the shedding capacity when assessing evidence at the activity level since the number of fibers transferred might be more influenced by the shedding than the activity, itself.

Concerning the action, fewer fibers were transferred in simulations involving little movement. The friction between the pillowcase and the participant partly explains why fewer fibers were more frequently recovered in night's sleep simulations compared to smothering simulations. Nevertheless, in some smothering experiments demonstrating little struggle, the number of fibers recovered was close to that observed in night's sleep simulations. This highlights a problematic situation for real case investigations. Since victims involved in homicides by smothering are commonly debilitated, disabled, very young or very old [28-30], they are consequently unable to effectively defend themselves. With little movement/friction involved, the number of fibers transferred is limited. Therefore, when dealing with a small number of textile fibers, interpretation should be approached with caution.

The distribution of fibers on the face was found to be relatively homogenous, independent of the activity (smothering or night's sleep) and pillowcase type. This unexpected result was attributed to the modus operandi and the amount of friction involved in the different simulations. Depending on the modus operandi used to simulate smothering, the pressure applied on the face, contact surface and the contact zones were variable. Thus, in simulations involving high pressure on the nose, more fibers were recovered in this area, as long as friction was involved. Due to the few number of simulations meeting these conditions, more experiments should be performed to investigate the impact of the modus operandi in greater detail. Hence, the nearly homogenous distributions observed in this study should not be used as a justification for avoiding the preservation of the localization, as it might be highly relevant for the case investigation.

Although respiratory tracts such as the nostrils and mouth were not investigated in this article, they are highly interesting and potentially relevant zones that should be studied in future research [31]. The transfer of fibers from other fabric sources such as duvets (duvet case) or towels - used as a way to smother someone - might also be investigated. Additionally, it has also been observed that some fibers were present in hair (near the face) - which could be studied in context of smothering and night sleep activities in the future. A study devoted to the secondary transfer of fibers from the head to pillowcases was, in fact, already carried out a few years ago [16] as well as a population study in human head hair [40].

Bayesian interpretation of the results was also performed for each pillowcase. The relative instability of the different models illustrates the problem with performing statistics on a limited number of simulations (25 for each type of activity and pillowcase). As previously mentioned, performing more simulations would not meet the operational requirements of a real investigation as they would be too time consuming and expensive in terms of material and human resources. Data analysis also highlighted that evidences outside the intervals covered by this article should be considered with caution. For such groups of fibers, extreme value analysis should be carried out in order to model the tails of the different distributions. This would generate high but hardly realistic likelihood ratios. Nevertheless, relevant information was gathered in terms of likelihood ratios showing that fiber evidence can, in fact, support smothering activity rather than legitimate night's sleep activity, or conversely - even with limited data.

Finally, this study shows that the probative value of a target fiber group (number and distribution) found on the face of a victim can be assessed at the activity level using (i) simulations performed in conditions close to those of criminal activities encountered by the investigation and (ii) the shedding capacity of the donor/source fabrics. 


\section{References}

[1] C.A. Pounds, K.W. Smalldon, The transfer of fibres between clothing materials during simulated contacts and their persistence during wear. Part I: fibre transference. Journal of the Forensic Science Society 15 (1) (1975) 17-27.

[2] R.R. Bresee, Evaluation of textile fiber evidence: A review. Journal of Forensic Sciences 32 (2) (1987) 510-521.

[3] M.C. Grieve, J. Dunlop, P.S. Haddock, Transfer experiments with acrylic fibres. Forensic Science International 40 (3) (1989) 267-277.

[4] C.B.M. Kidd, J. Robertson, The Transfer of Textile Fibres During Simulated Contacts. Journal of the Forensic Science Society 22 (3) (1982) 301-308.

[5] C.A. Pounds, K.W. Smalldon, The transfer of fibres between clothing materials during simulated contacts and their persistence during wear. Part III: A preliminary investigation of the mechanisms involved. Journal of the Forensic Science Society 15 (3) (1975) 197-207.

[6] J. Hellwig, The effect of textile construction on the shedding capacity of knitwear, 5th EFG Meeting, Berlin, Germany, 1997.

[7] H.G. Scott, The persistence of fibres transferred during contact of automobile carpets and clothing fabrics. Canadian Society of Forensic Science Journal 18 (4) (1985) 185-199.

[8] R. Cook, I.W. Evett, G. Jackson, P.J. Jones, J.A. Lambert, A model for case assessment and interpretation. Science \& Justice 38 (3) (1998) 151-156.

[9] C.G.G. Aitken, F. Taroni, Statistics and the evaluation of evidence for forensics scientists, 2nd ed., John Wiley and Sons, Ltd., Chichester, England, 2004.

[10] C. Champod, F. Taroni, Bayesian framework for the evaluation of fibre transfer evidence. Science \& Justice 37 (2) (1997) 75-83.

[11] C.A. Pounds, K.W. Smalldon, The transfer of fibres between clothing materials during simulated contacts and their persistence during wear. Part II: fibre persistence. Journal of the Forensic Science Society 15 (1) (1975) 29-37.

[12] J. Robertson, C. Roux, Transfer, Persistence and Recovery of Fibres, in: J. Robertson, M. Grieve (Eds.), Forensic examination of fibres, Taylor \& Francis, London, 1999, pp. 89-100.

[13] R. Palmer, Fibers: Transfer, Persistence, and Recovery, Wiley Encyclopedia of Forensic Science, John Wiley \& Sons, Ltd, 2009.

[14] R. Palmer, H.J. Burch, The population, transfer and persistence of fibres on the skin of living subjects. Science \& Justice 49 (4) (2009) 259-264.

[15] M.T. Salter, R. Cook, Transfer of fibres to head hair, their persistence and retrieval. Forensic Science International 81 (2-3) (1996) 211-221.

[16] R. Palmer, M. Banks, The secondary transfer of fibres from head hair. Science \& Justice 45 (3) (2005) 123-128.

[17] L. Lepot, T. Vanden Driessche, K. Lunstroot, F. Gason, K. De Wael, Fibre persistence on immersed garment-Influence of knitted recipient fabrics. Science \& Justice 55 (4) (2015) 248-253.

[18] C. Roux, J. Chable, P. Margot, Fibre transfer experiments onto car seats. Science \& Justice 36 (3) (1996) 143-151.

[19] M.C. Grieve, T.W. Biermann, Wool fibres - transfer to vinyl and leather vehicle seats and some observations on their secondary transfer. Science \& Justice 37 (1) (1997) 31-38.

[20] C. Roux, P. Margot, An attempt to assess the relevance of textile fibres recovered from car seats. Science \& Justice 37 (4) (1997) 225-230.

[21] R. Cook, C. Wilson, The significance of finding extraneous fibres in contact cases. Forensic Science International 32 (4) (1986) 267-273.

[22] A. Coxon, M. Grieve, J. Dunlop, A method of assessing the fibre shedding potential of fabrics. Journal of the Forensic Science Society 32 (2) (1992) 151-158.

[23] A.E. Parybyk, R.J. Lokan, A Study of the Numerical Distribution of Fibres Transferred From Blended Fabrics. Journal of the Forensic Science Society 26 (1) (1986) 61-68.

[24] M. Salter, R. Cook, A. Jackson, Differential shedding from blended fabrics. Forensic Science International 33 (3) (1987) 155-164. 
[25] J. Dix, Asphyxia (suffocation) and drowning, Color Atlas of Forensic Pathology, CRC Press LLC, 2000, pp. 98-115.

[26] C. Milroy, Asphyctic Deaths - Overview and Pathophysiology, in: J.A. Siegel, P.J. Saukko (Eds.), Vol. 3, Academic Press, Waltham, 2013, pp. 15-18.

[27] S. Ely, C. Hirsch, Asphyxial Deaths and Petechiae: A Review. Journal of Forensic Science 45 (6) (2000) 1274-1277.

[28] V.J.M. Di Maio, D.J. Di Maio, Asphyxia, Forensic Pathology, CRC Press, Boca Raton, 2001, pp. 229-277.

[29] S. Banaschak, P. Schmidt, B. Madea, Smothering of children older than 1 year of agediagnostic significance of morphological findings. Forensic Science International 134 (2-3) (2003) 163-168.

[30] J.A.J. Ferris, Asphyctic Deaths, in: J.A. Siegel, P.J. Saukko, G.C. Knupfer (Eds.), Encyclopedia of Forensic Sciences, Vol. 1-3, Academic Press, San Diego, 2000, pp. 308-316.

[31] B. National Institut of Criminalistic and Criminology (NICC, Survey on the recovery of fibres in smothering cases (June - August 2014), Internal Communication of the European Textile and Hair Group (ENFSI), 22nd EFG Meeting, Düsseldorf, Germany, 2014.

[32] K. De Wael, Fibres Intelligence - Linking Fibres Without A Source, 12th EFG Meeting, Prague, Czech Republic, 2004.

[33] T. Coyle, A. Larkin, K. Smith, S. Mayo, A. Chan, N. Hunt, Fibre mapping - a case study. Science \& Justice 44 (3) (2004) 179-186.

[34] K. Nehse, Using 1:1 Taping to reconstruct a source in: M.M. Houck (Ed.), Trace Evidence Analysis: More Cases in Mute Witnesses, Elsevier Academic Press, Italy, 2004, pp. 191-210.

[35] U. Decke, Advantages of 1:1 Topographies in High Profile Cases, 15th EFG Meeting, Madrid, Spain, 2007.

[36] K. De Wael, F. Gason, L. Lepot, K. Lunstroot, 10 years of 1:1 taping in Belgium - A selection of murder cases involving fibre examination. Science \& Justice 56 (1) (2016) 18-28.

[37] C. Champod, F. Taroni, The Bayesian Approach, in: J. Robertson, M. Grieve (Eds.), Forensic examination of fibres, Taylor \& Francis, London, 1999, pp. 379-398.

[38] F. Taroni, S. Bozza, A. Biedermann, P. Garbolino, C. Aitken, Data Analysis in Forensic Science: A Bayesian Decision Perspective, John Wiley \& Sons, 2010.

[39] K. De Wael, L. Lepot, K. Lunstroot, F. Gason, Evaluation of the shedding potential of textile materials. Science \& Justice 50 (4) (2010) 192-194.

[40] R. Palmer, S. Oliver, The population of coloured fibres in human head hair. Science \& justice: journal of the Forensic Science Society 44 (2) (2004) 83.

[41] J.-P. Beauthier, M. de Rood, Hypoxie et asphyxie, in: J.-P. Beauthier (Ed.), Traité de médecine légale, De Boeck, Italy, 2011, pp. 219-236.

[42] D. Neubert-Kirfel, Das Leitspurenkonzept: Der Dialog mit den stummen Zeugen der Tat. Kriminalistik 54 (6) (2000) 398-404.

[43] F. Monard Sermier, Etude des mécanismes de transfert des fibres en sciences forensiques, Institut de Police Scientifique, Faculté de Droit et des Sciences Criminelles, Université de Lausanne, 2007.

[44] N. Clayson, Fibres Finalise the Case, 12th EFG Meeting, Prague, Czech Republic, 2004.

[45] J. Jones, T. Coyle, Synthetic flock fibres: A population and target fibre study. Science \& Justice 51 (2) (2011) 68-71.

[46] K. Wiggins, P. Drummond, The analysis and comparison of blue wool fibre populations found at random on clothing. Science \& Justice 45 (3) (2005) 157-162.

[47] M.C. Grieve, T.W. Biermann, M. Davignon, The occurrence and individuality of orange and green cotton fibres. Science \& Justice 43 (1) (2003) 5-22.

[48] R. Cook, M.T. Webb-Salter, L. Marshall, The significance of fibres found in head hair. Forensic Science International 87 (2) (1997) 155-160.

[49] R. Palmer, V. Chinherende, A target fiber study using cinema and car seats as recipient items. Journal of Forensic Sciences 41 (5) (1996) 802-803. 
[50] R. Palmer, G. Polwarth, The persistence of fibres on skin in an outdoor deposition crime scene scenario. Science \&amp; Justice 51 (4) (2011) 187-189.

[51] J. Beirlant, Y. Goegebeur, J. Segers, J. Teugels, Statistics of Extremes: Theory and Applications, John Wiley \& Sons, Ltd, England, 2004.

[52] H. Penalva, M. Neves, S. Nunes, Topics in Data Analysis Using R in Extreme Value Theory. Metodolo`ski zvezki 10 (1) (2013) 17-29.

[53] R. Marquis, A. Biedermann, L. Cadola, C. Champod, L. Gueissaz, G. Massonnet, W.D. Mazzella, F. Taroni, T. Hicks, Discussion on how to implement a verbal scale in a forensic laboratory: Benefits, pitfalls and suggestions to avoid misunderstandings. Science \& Justice 56 (5) (2016) 364-370. 